\title{
RIESZ SETS AND THE RADON-NIKODYM PROPERTY
}

\author{
PATRICK N. DOWLING
}

(Received 21 June 1989)

Communicated by W. Moran

\begin{abstract}
Let $X$ be a complex Banach space, $G$ a compact abelian group and $\Lambda$ a subset of $\hat{G}$, the dual group of $G$. Then $L_{\Lambda}^{1}(G, X)$ has the Radon-Nikodym property if and only if $X$ has the Radon-Nikodym property and $\Lambda$ is a Riesz set. In particular, $H^{1}(\mathbb{T}, X)$ has the RadonNikodym property if and only if $X$ has the Radon-Nikodym property. This solves a problem of Hensgen.
\end{abstract}

1980 Mathematics subject classification (Amer. Math. Soc.) (1985 Revision): 46 B 22, 43 A 46. Keywords and phrases: Riesz sets, Radon-Nikodym Property.

\section{Introduction}

This note can be considered as a continuation of the work of Sundaresan [8] and an extension of a result of Lust-Piquard [6]. In [8] Sundaresan showed that if $(\Omega, \Sigma, \mu)$ is a finite measure space and $X$ is a Banach space then $L^{p}(\mu, X)$ has the Radon-Nikodym property if and only if $1<p<\infty$ and $X$ has the Radon-Nikodym property (see also [2] and [9]). On the other hand, Lust-Piquard [6] proved that if $G$ is a compact abelian group and if $\Lambda$ is a subset of $\hat{G}$, the dual group of $G$, then $L_{\Lambda}^{1}(G)$ has the Radon-Nikodym property if and only if $\Lambda$ is a Riesz set. In this note we will show that if $X$ is a complex Banach space then $L_{\Lambda}^{1}(G, X)$ has the Radon-Nikodym property if and only if $X$ has the Radon-Nikodym property and $\Lambda$ is a Riesz set. One consequence of this is that $H^{1}(\mathbb{T}, X)$ has the Radon-Nikodym property if and only if $X$ has the Radon-Nikodym property. This solves a problem of

(C) 1990 Australian Mathematical Society 0263-6115/90 \$A2.00+0.00 
Hensgen $[4,5]$. The method of proof we use here is very different from those of Sundaresan and Lust-Piquard. The idea of the proof stems from a method of Costé [1].

\section{Preliminaries and results}

Throughout this section, $G$ will denote a compact abelian group, $B(G)$ will denote the $\sigma$-algebra of Borel subsets of $G$ and $\lambda$ will denote normalised Haar measure on $G$. Let $\Gamma=\hat{G}$ be the dual group of $G$. If $\mu$ is a measure on $G$ and $\gamma \in \Gamma$ we define $\hat{\mu}(\gamma)$ by

$$
\hat{\mu}(\gamma)=\int_{G} \overline{\gamma(y)} d \mu(y) .
$$

A subset $\Lambda$ of $\Gamma$ is said to be a Riesz set if every Radon measure $\mu$ on $G$ which satisfies $\hat{\mu}(\gamma)=0$ for all $\gamma \notin \Lambda$ is absolutely continuous with respect to $\lambda$. If $G=\mathbb{T}$ then $\Gamma=\mathbb{Z}$ and by the $F$. and $M$. Riesz theorem $\mathbb{N}$ is a Riesz subset of $\mathbb{Z}$ (see [7]).

We define for a complex Banach space $X$, $L_{\Lambda}^{1}(G, X)=\left\{f \in L^{1}(G, X): \hat{f}(\gamma)=\int_{G} \overline{\gamma(y)} f(y) d \lambda(y)=0\right.$ for all $\left.\gamma \notin \Lambda\right\}$.

THEOREM. Let $G$ be a compact abelian group, let $\Lambda$ be a subset of $\hat{G}$ and let $X$ be a complex Banach space. Then $L_{\Lambda}^{1}(G, X)$ has the Radon-Nikodym property if and only if $X$ has the Radon-Nikodym property and $\Lambda$ is a Riesz set.

Proof. Suppose $L_{\Lambda}^{1}(G, X)$ has the Radon-Nikodym property. Then so does $X$ and $L_{\Lambda}^{1}(G)$, since they are isomorphic to subspaces of $L_{\Lambda}^{1}(G, X)$. By the result of Lust-Piquard [6], $\Lambda$ is a Riesz set because $L_{\Lambda}^{1}(G)$ has the Radon-Nikodym property.

Conversely, suppose $\Lambda$ is a Riesz set and $X$ has the Radon-Nikodym property. To prove that $L_{\Lambda}^{1}(G, X)$ has the Radon-Nikodym property it suffices to show that every bounded linear operator from $L^{1}[0,1]$ into $L_{\Lambda}^{1}(G, X)$ is Bochner representable [2]. To this end, let $T: L^{1}[0,1] \rightarrow$ $L_{\Lambda}^{1}(G, X)$ be a bounded linear operator and define

$$
F(A \times B)=\int_{B} T\left(1_{A}\right)(y) d \lambda(y)
$$

where $A$ is a Lebesgue measurable subset of $[0,1]$ and $B \in B(G)$. Let $C$ denote the algebra generated by the measurable rectangles of $[0,1] \times G$. It is clear that $F$ is a finitely additive bounded $X$-valued measure on $C$. 
For each $E \in \mathcal{C}$, we define $\nu(E)=|F|(E)$, the variation of $F$ on $E$. An elementary calculation shows that $\nu([0,1] \times G) \leq\|T\|<\infty$, so $\nu$ is finite. Therefore $\nu$ is a finitely additive non-negative real-valued measure on $C$ and $F$ is absolutely continuous with respect to $\nu$. Consequently $[2, \mathbf{p}$. 28 , Corollary 3 ], $F(C)$ is a relatively weakly compact subset of $X$. Hence, by $[2$, p. 27 , Theorem 2$], F$ has a unique countably additive extension to an $X$-valued measure, $\bar{F}$, on the $\sigma$-algebra generated by $C$. The $\sigma$-algebra generated by $C$ is the product $\sigma$-algebra $A \times B(G)$ where $A$ is the $\sigma$-algebra of Lebesgue measurable subsets of $[0,1]$.

We will show that the measure $\bar{F}: A \times B(G) \rightarrow X$ is absolutely continuous with respect to $m \times \lambda$, where $m$ is Lebesgue measure on $[0,1]$. To do this, it suffices to show that if $\left\{C_{n}\right\}_{n=1}^{\infty}$ is a sequence in $C$ such that $(m \times \lambda)\left(C_{n}\right) \rightarrow 0$ as $n \rightarrow \infty$, then $F\left(C_{n}\right) \rightarrow 0$ weakly in $X$ as $n \rightarrow \infty$. For $x^{*} \in X^{*}$ we define the bounded linear operator $T_{x^{*}}: L^{1}[0,1] \rightarrow L_{\Lambda}^{1}(G)$ by $\left(T_{x^{*}} f\right)(y)=$ $x^{*}((T f)(y))$, where $f \in L^{1}[0,1]$ and $y \in G$.

Since $\Lambda$ is a Riesz set, $L_{\Lambda}^{1}(G)$ has the Radon-Nikodym property [6] and so $T_{x^{*}}$ is Bochner representable. That is, there is a function

$$
g_{x^{*}} \in L^{\infty}\left([0,1], L_{\Lambda}^{1}(G)\right)
$$

such that

$$
T_{x^{*}} f=\int_{[0,1]} f(t) g_{x^{*}}(t) d m(t)
$$

for all $f \in L^{1}[0,1]$.

In particular, if $A \in A$ then

$$
T_{x^{*}}\left(1_{A}\right)=\int_{A} g_{x^{*}}(t) d m(t)
$$

Therefore, for $A \in A$ and $B \in B(G)$ we have

$$
\begin{aligned}
x^{*} F(A \times B) & =x^{*}\left(\int_{B} T\left(1_{A}\right)(y) d \lambda(y)\right) \\
& =\int_{B} x^{*}\left(T\left(1_{A}\right)(y)\right) d \lambda(y) \\
& =\int_{B}\left(T_{x^{*}}\left(1_{A}\right)\right)(y) d \lambda(y) \\
& =\int_{B}\left(\int_{A} g_{x^{*}}(t) d m(t)\right)(y) d \lambda(y) \\
& =\int_{B}\left(\int_{A}\left(g_{x^{*}}(t)\right)(y) d m(t)\right) d \lambda(y) \\
& =\int_{A \times B}\left(g_{x^{*}}(t)\right)(y) d(m \times \lambda)(t, y) .
\end{aligned}
$$


Note that,

$$
\begin{aligned}
\int_{[0,1]} \int_{G}\left|\left(g_{x^{*}}(t)\right)(y)\right| d \lambda(y) d m(t) & =\int_{[0,1]}\left\|g_{x^{*}}(t)\right\|_{L_{\Lambda}^{1}(G)} d m(t) \\
& =\left\|g_{x^{*}}\right\|_{L^{1}\left([0,1], L_{\Lambda}^{1}(G)\right)}<\infty
\end{aligned}
$$

Hence the function $\left.\left(g_{x^{*}}(\cdot)\right)(\cdot) \in L^{1}([0,1]) \times G\right)$. Therefore, if $(m \times \lambda)\left(C_{n}\right) \rightarrow$ 0 as $n \rightarrow \infty$ then $x^{*} F\left(C_{n}\right) \rightarrow 0$ as $n \rightarrow \infty$ and so we have proved that $\bar{F}$ is absolutely continuous with respect to $m \times \lambda$. Also, it is easily seen that $\bar{F}$ is a measure of bounded variation. Consequently, since $X$ has the Radon-Nikodym property there exists a function $g \in L^{1}([0,1] \times G, X)$ such that

$$
\bar{F}(C)=\int_{C} g(t, y) d(m \times \lambda)(t, y)
$$

for all $C \in A \times B(G)$.

By Fubini's theorem [3, p. 190], the function $g(t, \cdot)$ is an element of $L^{1}(G, X)$ for $m$-almost all $t \in[0,1]$. If we set

$$
H(t)= \begin{cases}g(t, \cdot) & \text { if } g(t, \cdot) \in L^{1}(G, X), \\ 0 & \text { otherwise, }\end{cases}
$$

then $H:[0,1] \rightarrow L^{1}(G, X)$ is an $m$-measurable function [3,p. 196, Lemma $16(\mathrm{~b})]$ and it is easy to see that $H \in L^{1}\left([0,1], L^{1}(G, X)\right)$. Fix $A \in A$. Then for all $B \in B(G)$

$$
F(A \times B)=\int_{A \times B} g(t, y) d(m \times \lambda)(t, y)=\int_{B}\left(\int_{A} g(t, y) d m(t)\right) d \lambda(y) .
$$

Therefore

$$
\int_{B}\left(\int_{A} g(t, y) d m(t)-T\left(1_{A}\right)(y)\right) d \lambda(y)=0
$$

for all $B \in B(G)$. Thus

$$
T\left(1_{A}\right)(y)=\int_{A} g(t, y) d m(t)
$$

for $\lambda$-almost all $y \in G$, and so as elements of $L^{1}(G, X)$

$$
T\left(1_{A}\right)=\int_{A} g(t, \cdot) d m(t)=\int_{A} H(t) d m(t) .
$$

Since $T$ is $L_{\Lambda}^{1}(G, X)$-valued so is $H, m$-almost surely. We define $\tilde{H}:[0,1]$ $\rightarrow L_{\Lambda}^{1}(G, X)$ by

$$
\tilde{H}(t)= \begin{cases}H(t) & \text { if } H(t) \in L_{\Lambda}^{1}(G, X) \\ 0 & \text { otherwise }\end{cases}
$$


Then for all $A \in A, T\left(1_{A}\right)=\int_{A} \tilde{H}(t) d m(t)$ and $\tilde{H} \in L^{1}\left([0,1], L_{\Lambda}^{1}(G, X)\right)$. Hence, by $[2$, p. 62 , Lemma 4] $T$ is Bochner representable. This completes the proof.

If we let $G=\mathbb{T}$ then $\hat{G}=\mathbb{Z}$. We define the Hardy space $H^{1}(\mathbb{T}, X)$, for a complex Banach space $X$, by

$$
H^{1}(\mathbb{T}, X)=\left\{f \in L^{1}(\mathbb{T}, X): \hat{f}(n)=0 \text { for all } n<0\right\} .
$$

In [4] Hensgen proves that if $X$ is a separable dual space then $H^{1}(\mathbb{T}, X)$ is also a separable dual and so has the Radon-Nikodym property, and asks the question; If $X$ has the Radon-Nikodym property does $H^{1}(\mathbb{T}, X)$ have the Radon-Nikodym property?

We give a positive answer to this question.

COROLlaRY. If $X$ is a complex Banach space with the Radon-Nikodym property then $H^{1}(\mathbb{T}, X)$ has the Radon-Nikodym property.

Proof. $H^{1}(\mathbb{T}, X)=L_{\Lambda}^{1}(G, X)$ where $\Lambda=\{n \in \mathbb{Z} ; n \geq 0\}$. By the $\mathrm{F}$. and M. Riesz theorem [7] $\Lambda$ is a Riesz subset of $\mathbb{Z}$. An application of the theorem finishes the proof.

\section{Acknowledgements}

I would like to thank Elias Saab for pointing out an error in my original proof of the theorem.

\section{References}

[1] A. Costé, 'Sur les operateurs representables', C. R. Acad. Sci. Paris 298 (1984), 205-208.

[2] J. Diestel and J. J. Uhl, Vector measures, (Math. Surveys, no. 15, Amer. Math. Soc., Providence, R.I., 1977).

[3] N. Dunford and J. T. Schwartz, Linear operators, Part I, (Interscience Publishers, New York, 1957).

[4] W. Hensgen, 'Operatoren $H^{1} \rightarrow X$ ', Manuscripta Math. 59 (1987), 399-422.

[5] W. Hensgen, Hardy-Räume vektorwertiger funktionen, (Dissertation, Munich, 1986).

[6] F. Lust-Piquard, 'Ensembles de Rosenthal et ensembles de Riesz', C. R. Acad. Sci. Paris 282 (1976), A833-835.

[7] W. Rudin, Fourier analysis on groups, (Tracts in Mathematics, no. 12, 1962). 
[8] K. Sundaresan, 'The Radon-Nikodym property in Lebesgue-Bochner function spaces', J. Funct. Anal. 24 (1977), 276-279.

[9] J. B. Turett and J. J. Uhl, ' $L_{p}(\mu, X)(1<p<\infty)$ has the Radon-Nikodym property if $X$ does by martingales', Proc. Amer. Math. Soc. 61 (1976), 347-350.

\author{
Miami University \\ Oxford, Ohio 45056 \\ U.S.A.
}

